\title{
Correction to: Perso2U: Exploration of User Emotional States to Drive Interface Adaptation
}

\author{
Julián Andrés Galindo
}

\section{Correction to:}

Chapter "Perso2U: Exploration of User Emotional

States to Drive Interface Adaptation" in:

M. Botto-Tobar et al. (Eds.): Technology Trends, CCIS 895, https://doi.org/10.1007/978-3-030-05532-5_21

The chapter was inadvertently published with three author names. This information has been updated later at the request of the corresponding author with removal of the second and third author names with their consent to the initially published version of chapter "Perso2U: Exploration of User Emotional States to Drive Interface Adaptation". 